\title{
ON THE ANOMALOUS OPTICAL PROPERTIES OF HEIKOLITE*
}

\author{
TOKUGORO SHODA
}

Institute of Mineralogy, Faculty of Science, University of Tokyo

As is widely known certain alkali amphiboles ${ }^{15)(1011) 12 / 13 / 14}$ are anomalous in that they do not show extinction between crossed nicols. An example of such amphiboles is heikolite, a dark green prismatic amphibole. It was found by $\mathrm{Y}$. Kinosaki ${ }^{9}$ in pegmatite in Heikomen, ${ }^{* *}$ Korea.

Z. Harada2' gave it the formula; $(\mathrm{OH})_{2}(\mathrm{Na}, \mathrm{K}, \mathrm{Ca}, \mathrm{Mn})(\mathrm{Mg}, \mathrm{Fe}$, $\mathrm{Fe}, \mathrm{Ti}, \mathrm{Al})_{5}(\mathrm{Si}, \mathrm{Al})_{8} \mathrm{O}_{22}$ on the basis of a chemical analysis by $\mathrm{S}$. Komatsu and concluded that the mineral is a variety of alkali amphibole containing approximately $30 \%$ glaucophane and $70 \%$ riebeckite molecules. The optical properties, too, of heikolite have been studied by several investigators.932/3)

The most salient characteristics of this mineral is that it does not show on (010) section extinction between crossed nicols with the monochromatic light.

The writer will give below an account of a study of this mineral undertaken to know more about these extraordinary interesting phenomena.

\section{Experimental}

As heikolite shows very strong dispersion, it is desirable to employ monochromatic lights of various wave lengths. For this

* Read at the meeting of the Mineralogical Society of Japan on October 4, 1953.

** Hoam-san, P'yŏnggang-myŏn, P'yŏnggang-gun, Kangwŏn-do, Korea.

朝鲜江原道平康郡平康面虎岩山。 
purpose, a monochromator was set between the light source and the microscope.

In the spectrum emitted by a helium lamp, the lines at 668,587 , $501,468 \mu \mu$ can be used. However, as the intensity of these lines was considerably weak and, moreover, it was not easy to stabilize the source fed by alternating current, an eight-volt lamp fed by a battery was used together with a monochromator to obtain the monochromatic light.

The intensity of transmitted monochromatic light was measured by a photometer ${ }^{7)}$ which consists of a photomultiplier (RCA 931A) and a galvanometer.

Measurements were made on a relative scale.

\section{Theoretical consideration}

Let $A$ be the amplitude of light wave passing through the polarizer whose vibration direction is represented by OP in Fig. 1;

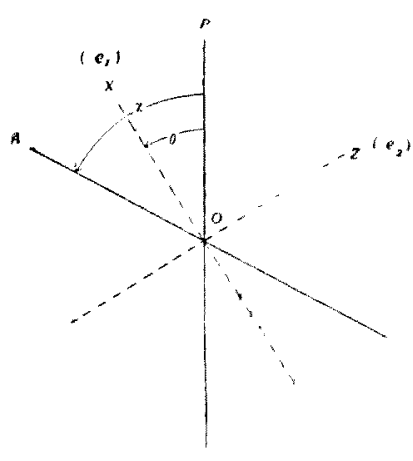

Fig. 1. Diagram showing the vibra. tion direction of light through two nicol prisms and a thin section. upon entering a thin section with vibration direction $O X$ and $O Z$, it is resolved into two components whose amplitudes are $A \cos \theta$ and $A \sin \theta$ respectively. If these two components pass through the thin section so as to have, at emergence, the phase difference $\Delta$ and the absorption ratio $e_{1}, e_{2}$, they will be brought back to the same vibration direction of the analyzer which is represented by OA. The intensity of the resultant of two vibrations which have the amplitude $A e_{a} e_{1} \cos \theta \cos (\chi-\theta)$ and $A e_{a} e_{2} \sin \theta \sin (\chi-\theta)$, when $e_{a}$ is the reduction ratio of amplitude by absorption or reflection through the analyzer and the other optical parts, is given by 


$$
\begin{array}{r}
I=e_{a}^{2} A^{2}\left\{e_{1}^{1} \cos ^{2} \theta \cos ^{2}(\chi-\theta)+e_{2}^{2} \sin ^{2} \theta \sin ^{2}(\chi-\theta)\right. \\
\left.-2 e_{1} e_{2} \cos \Delta \cos \theta \sin \theta \cos (\chi-\theta) \sin (\chi-\theta)\right\}
\end{array}
$$

The values of $e_{1}, e_{2}$ and $\Delta$ are peculiar to a section. Therefore, rotating the section, we obtain a curve of intensity variation with $\theta$ for a definite value of $\chi$.

In the case of transparent colorless mineral, we can put $e_{1}=e_{2}=1$ in the equation above. Therefore, the theoretical intensity curve is symmetrical with respect to the positions with $\theta=\frac{\chi}{2} \pm n \frac{\pi}{2}$ (maximum positions) and those with $\theta=\frac{\chi}{2} \pm \frac{\pi}{4} \pm n \frac{\pi}{2}$ (minimum positions) where $n=0,1,2 \ldots$ In addition to this, it can be shown that the difference between the maximum and the minimum for the same value of $\chi$ is kept constant ( $=e^{2} a A^{2} \sin ^{2} \frac{4}{2}$ ) regardless of the value of $\chi$ and that the sum of the value of the intensity for $\chi=\frac{\pi}{2}$ and that for $\chi=0$ is $e_{a}^{2} A^{2}$ for the equal value of $\theta$.

In the case of pleochroic mineral, the curve is symmetrical with respect to the positions with $\theta=\frac{\chi}{2} \pm n \frac{\pi}{2}$, but the maximum and the minimum values and their positions vary with the values of $e_{1}, e_{2}, \Delta$ and $\chi$.

Substituting $-\chi$ for $\chi$, and $\theta-\chi$ for $\theta$ in the equation above, we have the same equation; that is, the phase difference between the curve for $\chi$ and that for $-\chi$ is $\chi$.

\section{Preliminary experiments with standard minerals}

(1) Topaz. The (010) section of topaz from the Ebisu Mine, Gifu Prefecture was examined as an example of colorless mineral using the light of $\lambda=668 \mu \mu$.

For each value of $\chi$, the intensity curve showed the same behaviour at every $\frac{\pi}{2}$ of the angle of rotation and the symmetrical behaviour with respect to the positions of maxima and minima.

The observed intensities which are shown as curves (a), (b) and (c) in Fig. 2 are practically in agreement with those calculated. 


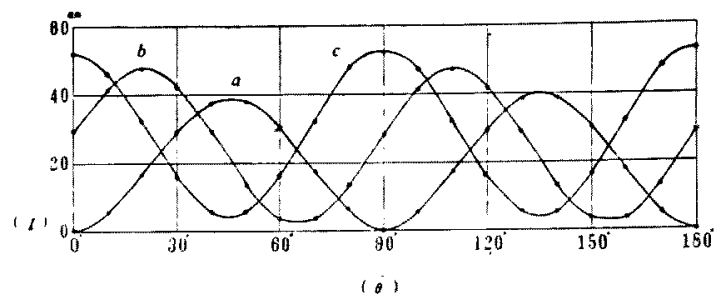

Fig. 2. Intensity variations for the (010) section of topas.
$(\lambda=668 \mu \mu).(a) \chi=90^{\circ}$
(b) $x=40^{3}$
(c) $x=0^{\circ}$

(2) Aegirine. The (010) section of aegirine from Kakubuzan, $\left.{ }^{* 9}\right) 8$ ) Korea was observed as an example of pleochroic mineral with the light of $\lambda=587 \mu \mu$ and the intensity was compared with that calculated from the equation.

The values of $e_{1}, e_{2}$ and $\Delta$ for this section were determined as follows.

The scale readings on the apparatus with parallel nicols and without section was found to be $e^{2} a A^{2}=275 \mathrm{~mm}$. Similarly, for $\chi=0$ and $\theta=0$ we have $e_{a}^{2} e^{2} A^{2}=8 \mathrm{~mm}$., for $\chi=0$ and $\theta=\frac{\pi}{2}$. $e^{2}{ }_{a} e_{2}^{2} A^{2}=54 \mathrm{~mm}$., for $\chi=\frac{\pi}{2}$ and $\theta=\frac{\pi}{4}$. $\quad \frac{1}{4} e^{2} a A^{2}\left(e^{2}{ }_{1}+e^{2}{ }_{2}-2 e_{1} e_{2} \cos \Delta\right)=21 \mathrm{~mm}$, and for $\chi=0$ and $\theta=\frac{\pi}{4} \quad \frac{1}{4} e^{2} A^{2}\left(e^{2}{ }_{1}+e^{2}{ }_{2}+2 e_{1} e_{2} \cos A\right)=10 \mathrm{~mm}$.

Assuming that the scale readings on the apparatus is proportional to the intensity to be measured and that $e^{2} a A^{2}=1$, we obtain the values of $e_{1}, e_{2}$ and $\Delta$ as follows.

$$
e_{1}=0.170 \quad e_{2}=0.443 \quad \Delta=121^{\circ} 57^{\prime}
$$

Fig. 3 shows the calculated and the observed values of intensity for $\chi=80^{\circ}$, where the broken line represents the values calculated using the above values of $e_{1}, e_{2}$ and $\Delta$, and the full line, the corresponding observed values.

* Hangmu-san, Ch'unhung-dong, Yangsa-myŏn, Kilchu-gun, Hamgyŏng-bukto. 咸鏡北道吉州郡暘社面春與洞鶴舜山。 
The observed positions of the maxima and the minima determined from the curves for various values of $\chi$ are shown as dots in Fig. 4 together with calculated positions which is shown as broken lines. Of the two maxima shown in Fig. 4 and the following figures, $\operatorname{Max}_{1}$ denotes the one with the larger value and of two minima $\mathrm{Min}_{1}$ denotes the one with the smaller value.

Each measurement for a definite value of $\chi$ was made under the different condition. Therefore, in order to see the variation of the values of maxima and minima, the measurement of the intensity under the same condition was made. The results are shown in Fig. 5 together with obs. calc.

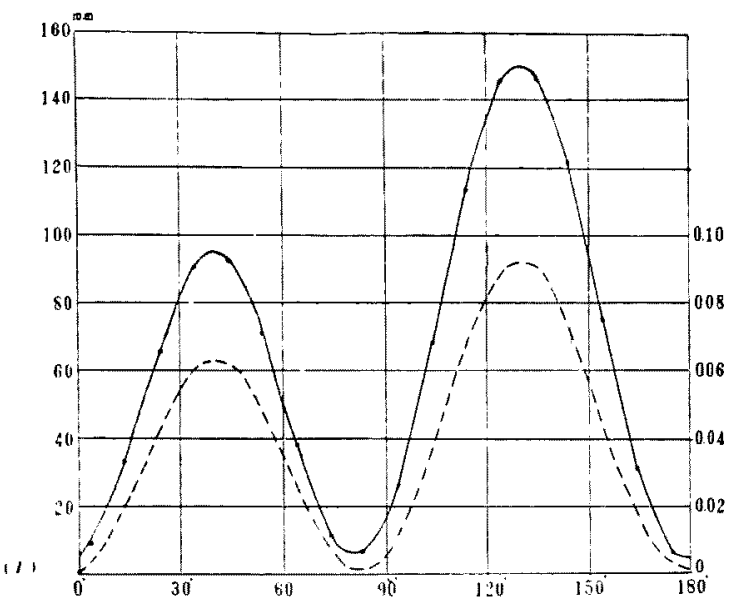

Fig. 3. Intensity variations for the (010) section of aegirine. $\left(\chi=80^{\circ}, \lambda=587 \mu \mu.\right)$ Full line: observed. Broken line: calculated.

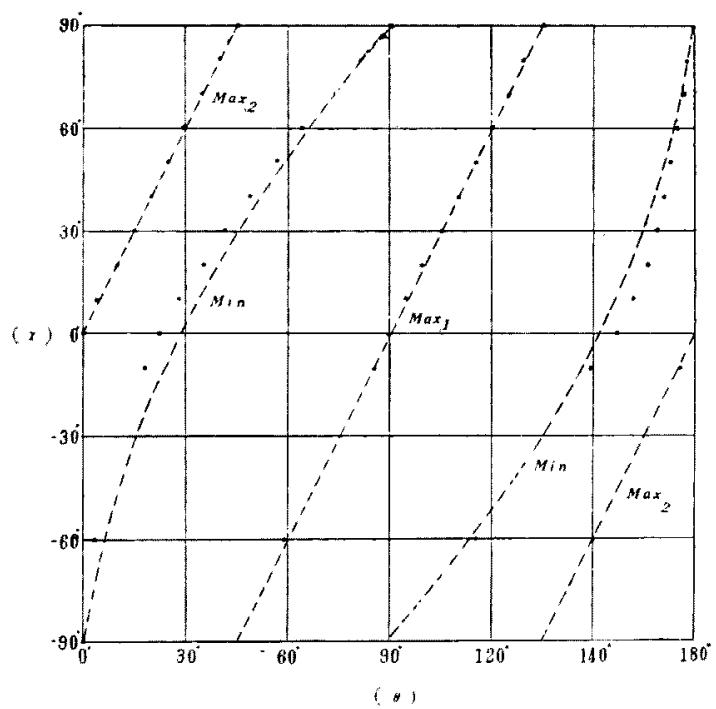

Fig. 4. Positions of maximum and minimum intensity for the (010) section of aegirine. $(\lambda=587 \mu . \mu$.$) Dots : observed. Broken$ lines: calculated. 


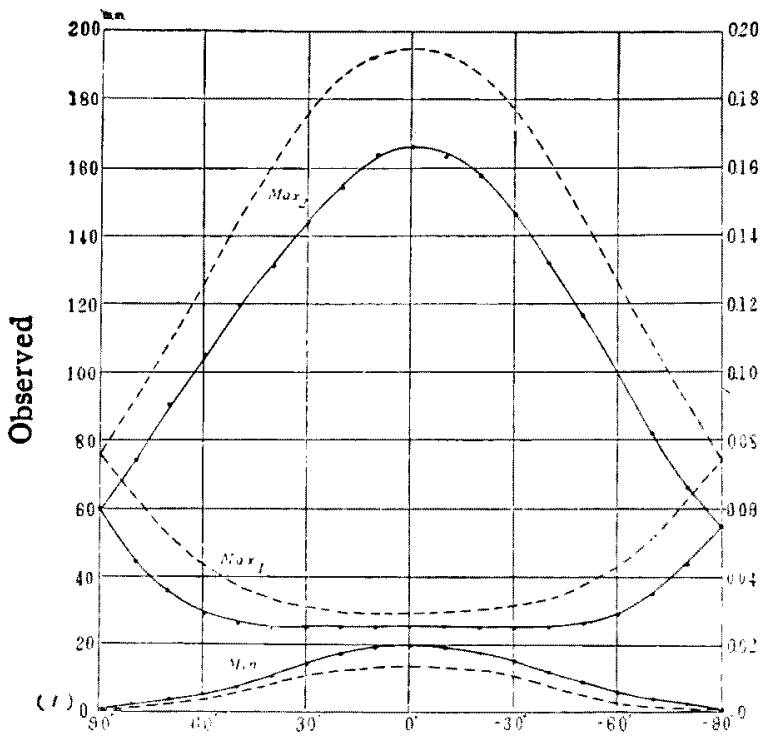

Fig. 5. Variation of the values of maximum and minimum intensity for the (010) section of aegirine. $(\lambda=587 \mu$. $)$ Dots and full lines: observed. Broken lines: calculated.

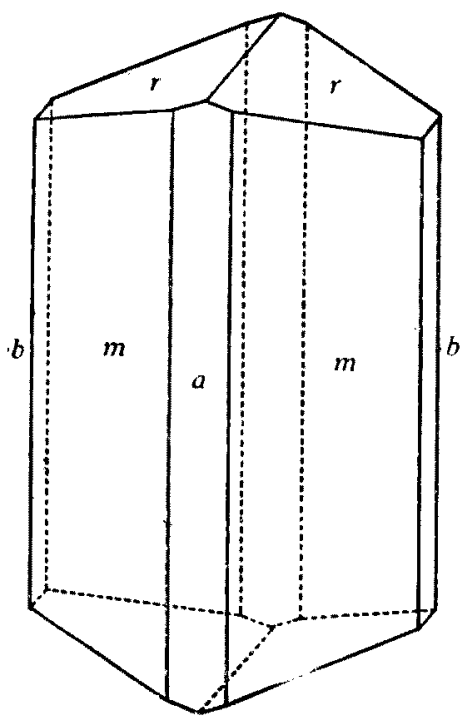

Fig. 6. Crystal form of heikolite from Kansenri having holohedral habit. the calculated values.

As evident in Figs. 3,4 and 5 , the relative intensities observed are in good accordance with the calculated values.

\section{Measurements on heikolite}

The specimen used in this experiment is one from Kansenri, ${ }^{* 9}$ ) in the crystal form $m(110), r(011), p(\overline{101})$, $a(100)$ and $b(010)$ as shown in Figs. 6 and 7. The result of goniometry is shown in Table 1.

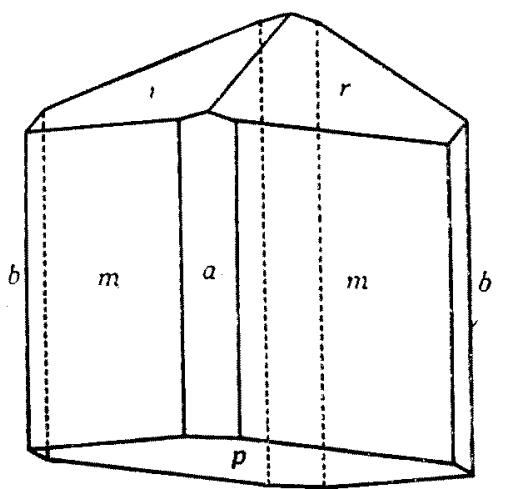

Fig. 7. Crystal form of heikolite from Kansenri having hemimorphic habit.

* Kŏnchh'ŏn-ni, Kŭn-bung-myŏn, Kŭmhwa-gun, Kangwŏn-do, Korea. 朝能江原道金化郡近北面㪍川里 
Table 1.

\begin{tabular}{|c|c|c|c|c|c|}
\hline \multirow{2}{*}{ Form } & \multicolumn{2}{|c|}{ Mean observed value } & \multicolumn{2}{|c|}{ Calculated value** } & \multirow{2}{*}{$\mathrm{n}^{* * *}$} \\
\hline & $p$ & $\varphi$ & $p$ & 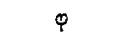 & \\
\hline$a(100)$ & $00^{\circ} 00^{\prime}$ & $90^{\circ} 24^{\prime}$ & $90^{\circ} 00^{\prime}$ & $90^{\circ} 00^{\prime}$ & 3 \\
\hline$b(010)$ & $90 \quad 02$ & $010^{*}$ & $90 \quad 00$ & $0 \quad 00$ & 5 \\
\hline$m(110)$ & $90 \quad 00$ & $\begin{array}{ll}62 & 17\end{array}$ & $90 \quad 00$ & $62 \quad 05$ & 12 \\
\hline$r(011)$ & $22 \quad 53$ & $45 \quad 51$ & $22 \quad 20$ & $44 \quad 21$ & 4 \\
\hline$p(\overline{1} 01)$ & $14 \quad 18$ & $-90 \quad 43$ & $14 \quad 58$ & $-90 \quad 00$ & 2 \\
\hline
\end{tabular}

* Origin.

** After Goldschmidt's "Winkeltabellen "

*** Number of face measured.

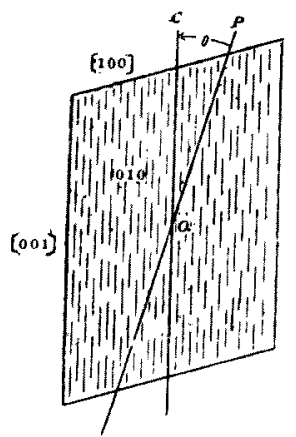

Fig. 8. (010) section of heikolite. OP indicates the vibration direction of polarizer.

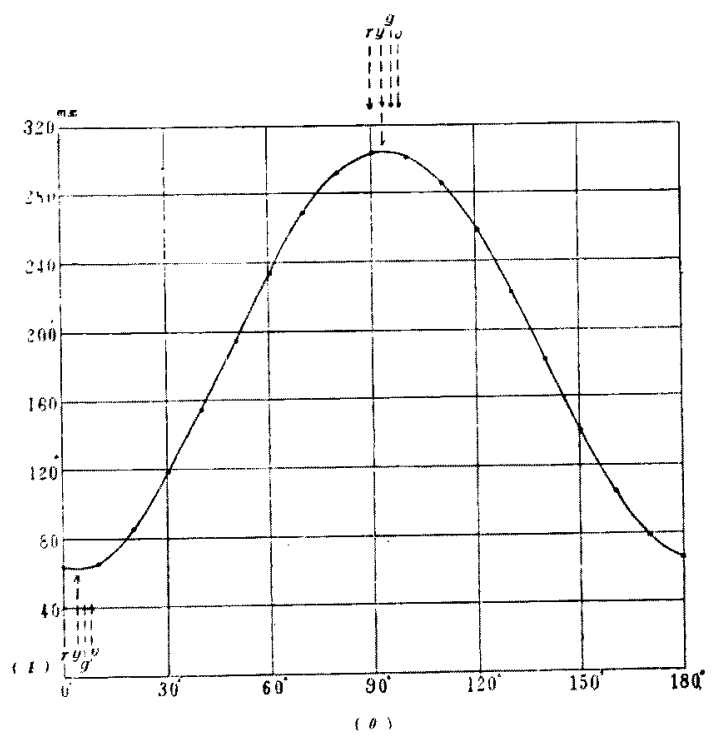

Fig. 9. Intensity variation for the (010) section of heikolite with the polarizer only. $(\lambda=587 \mu \mu$. $)$ Arrows show the positions of the maxima and the minima with various wave lengths. $\mathrm{r}-668 \mu . \quad \mathrm{g}-587 \mu . . \quad \mathrm{g}-501 \mu . . \quad \mathrm{v}-468 \mu \mu$.

The intensity measured through the (010) section of heikolite with the polarizer only, show the maximum value near the position at which the (110) cleavage trace in the section is perpendicular to the vibration direction of the polarizer. $\left(\theta \doteqdot \frac{\pi}{2}\right.$ in Fig. 8) However, 
there occurs slight dispersion of the position of the maximum and the minimum intensity. The curve of the intensity variation for $587 \mu \mu$ is shown in Fig. 9. In this figure, the positions of the maxima and the minima obtained with lights of different wave lengths are also shown.

The curves obtained from the data measured with crossed nicols showed the same behaviour at every $\frac{\pi}{2}$ of the angle of rotation and the symmetrical behaviour with respect to the positions of maxima and minima. However, these positions are shifted considerably with wave length. The curve obtained from the measurement with $587 \mu \mu$ is shown in Fig. 10 in which the variation of the positions of maxima and minima with wave length is also shown. It is to be noted that the minimum intensity is considerably strong.

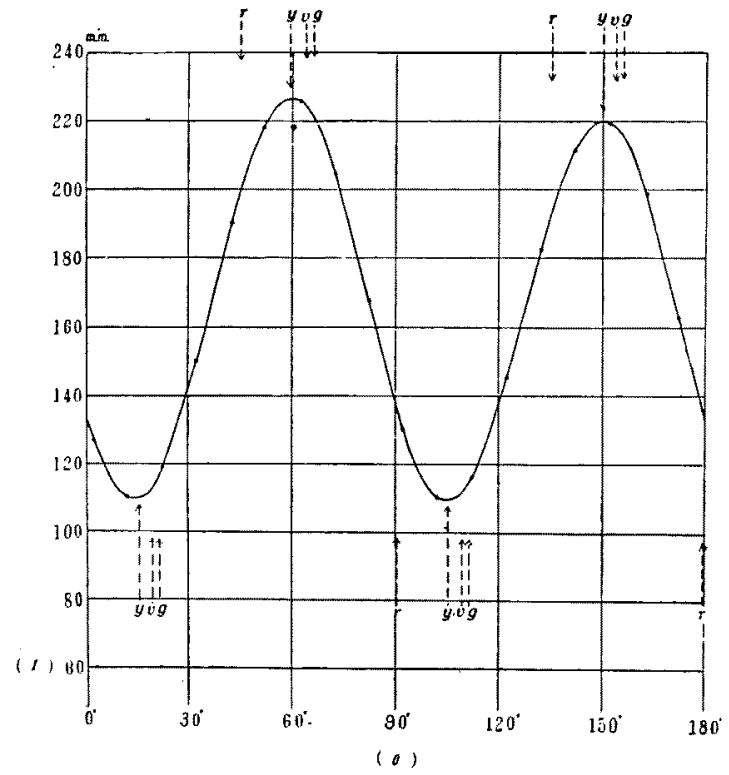

Fig. 10. Intensity variation for the (010) section of heikolite with crossed nicols. $(\lambda=587 \mu$.) Arrows show the positions of the maxima and the minima with various wave lengths. $\mathrm{r}-668 \mu \mu . \mathrm{y}-587 \mu \mu . \mathrm{g}-501 \mu \mu . \mathrm{v}-468 \mu \mu$.
For the measurement with oblique nicols, with $587 \mu \mu$, $501 \mu \mu$ or $468 \mu \mu$, we obtained only an asymmetrical curve, but not such a symmetrical one as in the case of aegirine. As shown in Fig. 3, there are two maxima with different values separated at a distance of $\frac{\pi}{2}$ and two minima with same values symmetrically situated with respect to the positions of the maxima between $\theta=0$ and $\pi$. However, in 
the case of heikolite, there are two different maxima and two different minima for the larger values of $\chi$, and only one minimum and maximum for the smaller values of $\chi$.

Fig. 11 shows the curve for $\chi=60^{\circ}$ and $\lambda=501 \mu \mu$, and Fig. 12 shows that for $\chi=0^{\circ}$ and $\lambda=501 \mu \mu$.

For $668 \mu \mu$, the shape of the intensity curve is slightly different from those for other wave lengths. In this case, as shown in Figs. 13 and 14, the curve shows the behaviour simillar to that of aegirine.

Figs. 15, 16 and 17 show the positions of $\operatorname{Max}_{1}, \operatorname{Max}_{2}, \operatorname{Min}_{1}$ and $\mathrm{Min}_{2}$ for different values of $\chi$. From these figures, it will be seen that the angle of $\theta$ corresponding to the maximum of the

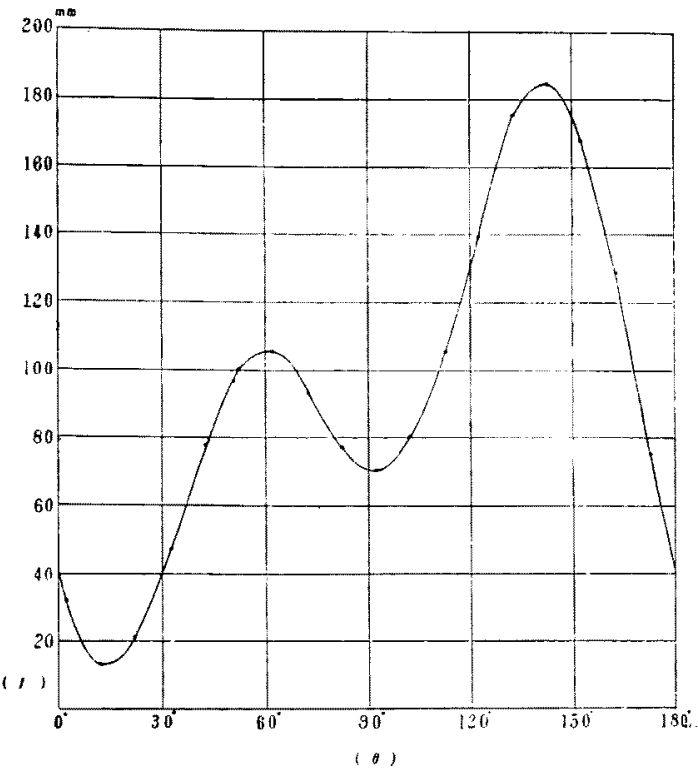

Wig. 11. Intensity variation for the $(010)$ section of heikolite. $\left(x=60^{\circ} \quad x=501 \mu u.\right)$

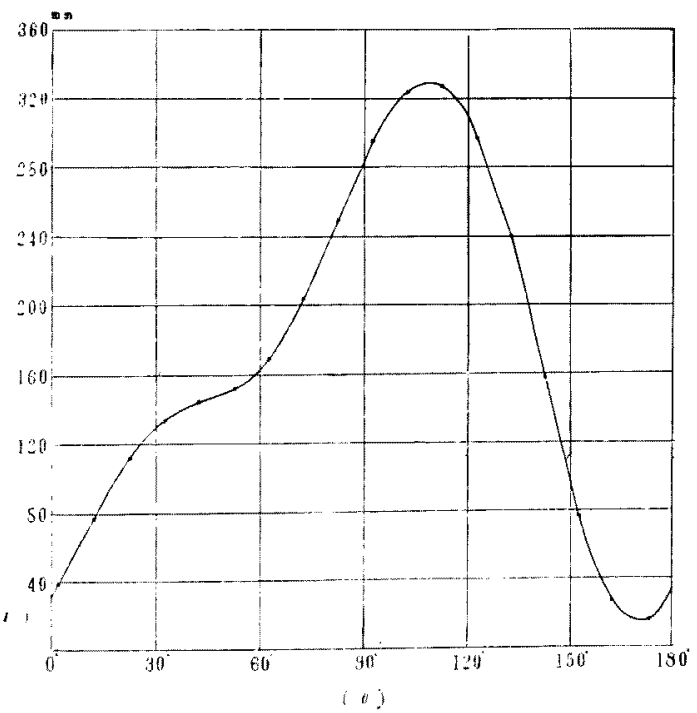

Fig. 12. Intensity : variation for the (010) section of heikolite. $\left(x=0^{\circ}, \lambda=501\right.$ p.p. $)$ 
curve for $\chi$ is different by $\chi$ from that for $-\chi$.

The diagram for the variation of the values of $\operatorname{Max}_{1}, \operatorname{Max}_{2}, \operatorname{Min}_{1}$ and $\mathrm{Min}_{2}$ which were measured by the same method as in the case of aegirine are shown in Figs. 18, 19 and 20. In these figures, it will be seen that for $587 \mu \mu$ the second maximum-Max disappear $^{-}$

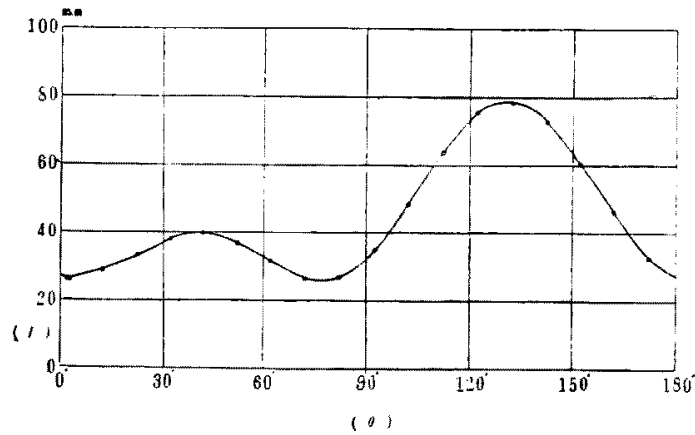

Fig. 13. Intensity variation for the (010) section of heikolite, $\left(x=80^{\circ}, \lambda=668 \mu\right.$... $)$

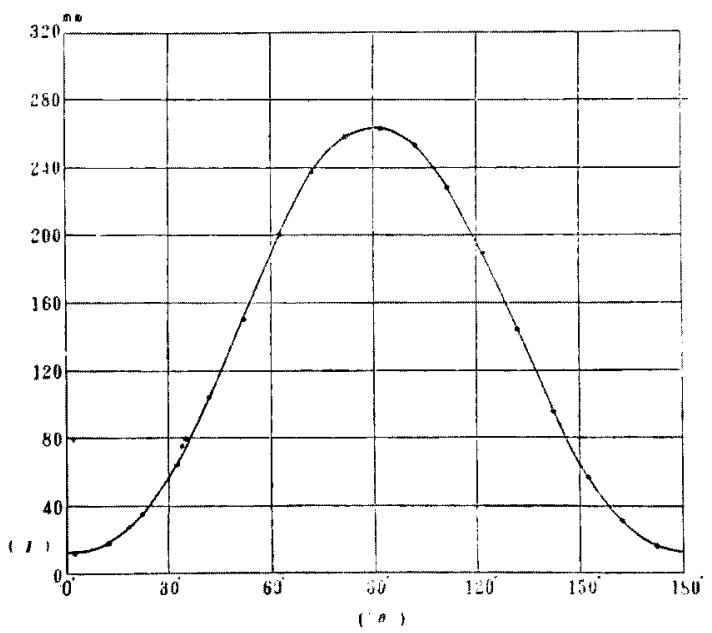

Fig. 14. Intensity variation for the (010) section of heikolite, $\left(x=0^{\circ}, \lambda=668\right.$ ust. $)$ at the value of $\chi \doteqdot$ $70^{\circ}$, while that for $468 \mu \mu$ it can be observed down to $\chi$ $\doteqdot 40^{\circ}$.

One might consider that the asymmetry of these curves is not due to the optical property of the $\mathrm{mi}$ neral itself, but due to the deviation of the optical axis of the microscope from the normal of the section. However, this has been shown not to be the case by turning the section inside out and observing it in the direction of $[0 \overline{1} 0]$ and rotating the stage towards the opposite direction.

The values of the intensity measured for $[0 \overline{1} 0]$ and $\chi=80^{\circ}$ are represented by 
curve (b) in Fig. 21, in which curve (a) corresponding to those for [010] and $\chi=$ $-80^{\circ}$ is shown for the sake of comparison. It is readily seen that curve (a) runs parallel with curve (b) and that the positions of the maxima and the minima of curve (a) coincide exactly with those of curve (b). Therefore, there is no appreciable deviation of the direction of the optical axis from that of the normal.

According to the photoelectrical measurement by $\mathrm{S}$. Hori, ${ }^{3)}$ the values of intensity observed for this mineral were in good agreement with those calculated on the basis of his assumption that this mineral consists of two parts of different orientations. However, the intensity

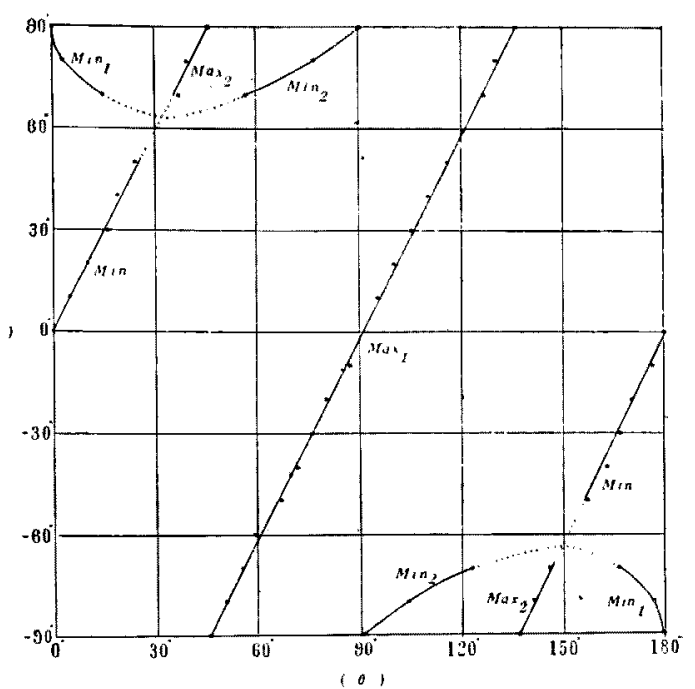

Fig. 15. Positions of maximum and minimum intensity for the (010) section of heikolite.

$$
\left(\lambda_{1}=668 \text { u.u. }\right)
$$

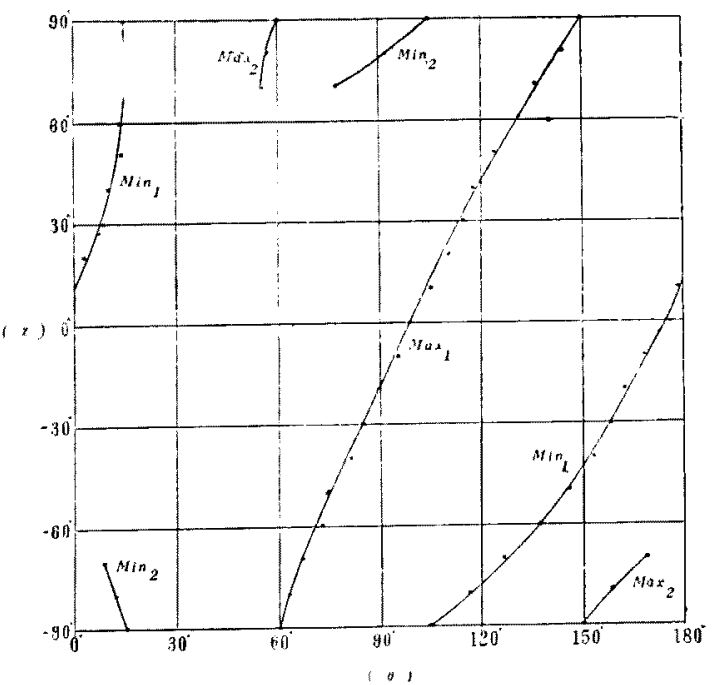

Fig. 16. Positions of maximum and minimum intensity for the (010) section of heikolite.

$$
(\lambda=587 \text { u... })
$$




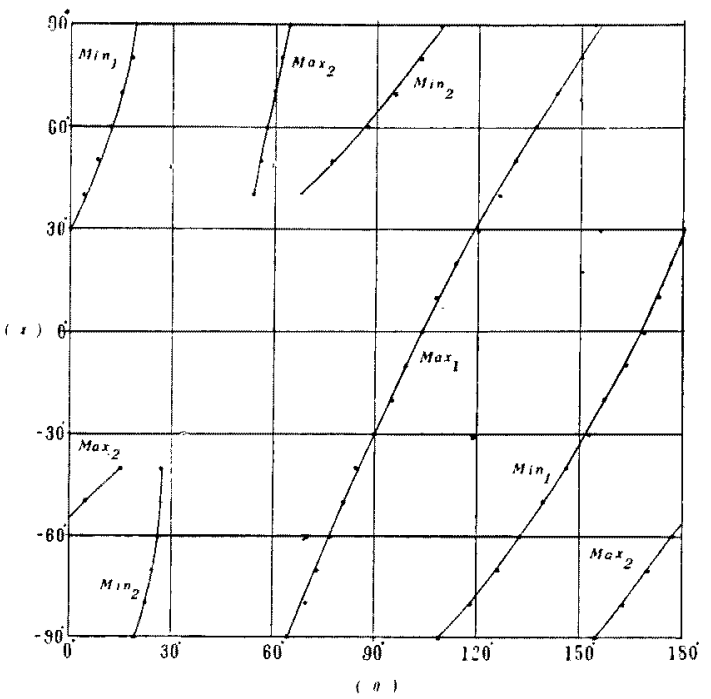

Fig. 17. Positions of maximum and minimum intensity for the (010) section of heikolite.

$$
(\lambda=468 \text { y.u. })
$$

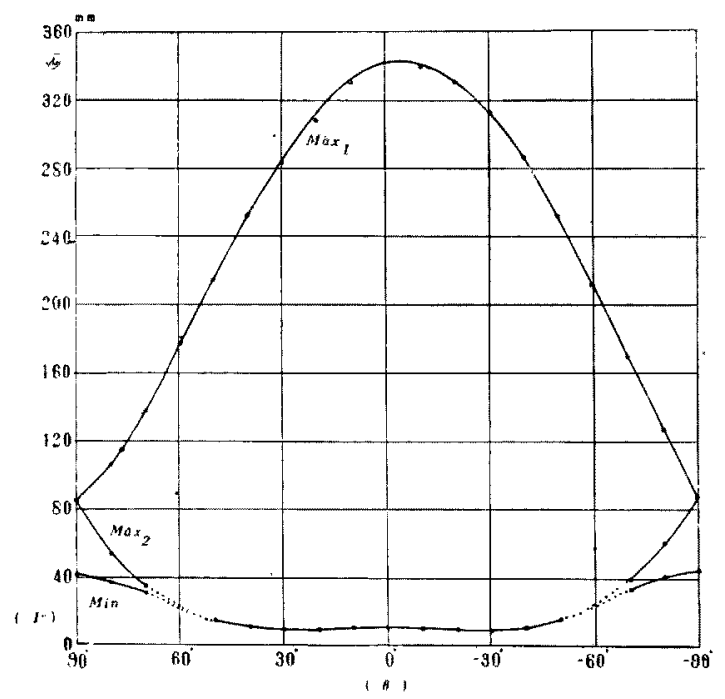

Fig. 18. Variation of the values of maximum and minimum intensity for the (010) section of heikolite. $\left(\lambda=668 \mu, \mu_{.}\right)$ 


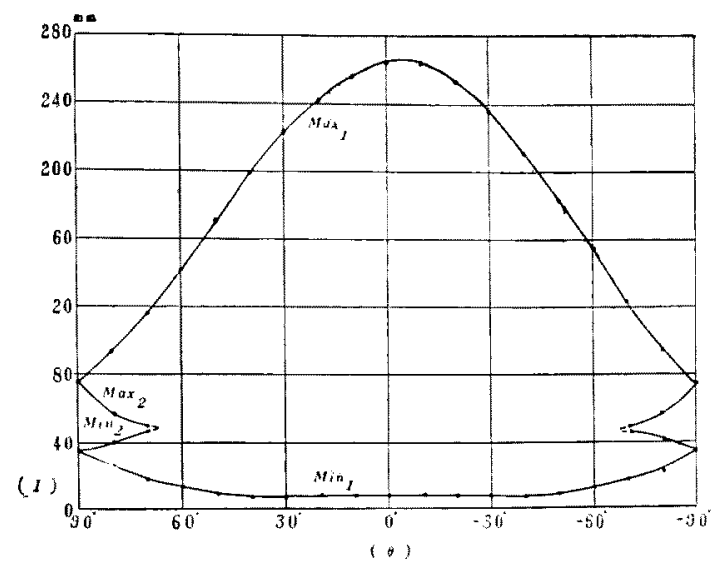

Fig. 19. Variation of the values of maximum and minimum intensity for the (010) section of heikolite. $(\lambda=587 \mu$. $)$

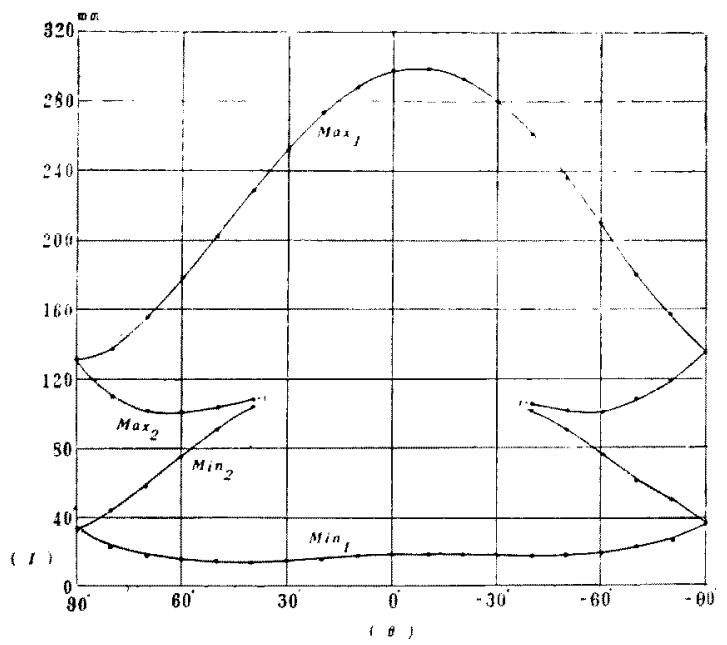

Fig. 20. Variation of the values of maximum and minimum intensity for the (010) section of heikolite. $(\lambda=468$ u. .) 


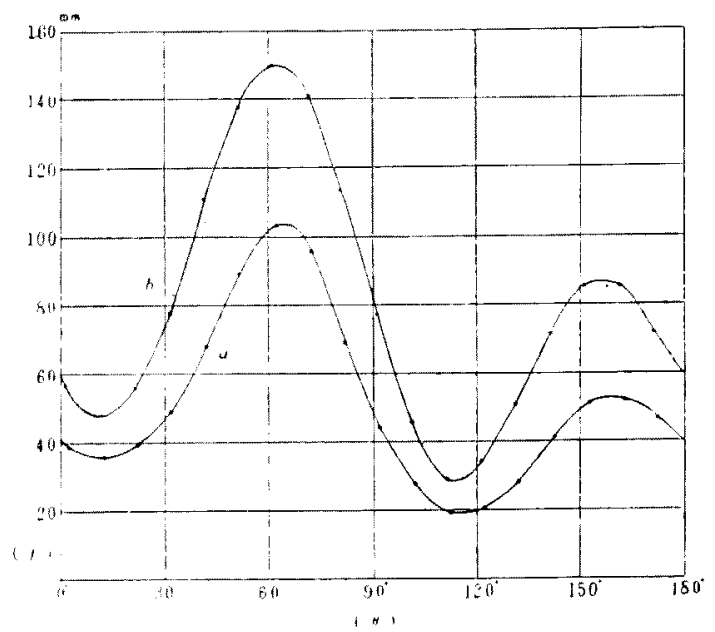

Fig. 21. Intensity variations for the (010) section of heikolite. $(\lambda=587$ y.u. $)$

(a) Observed in the direction of [010], for $\chi=-80^{\circ}$.

(b) Observed in the direction of $[010]$, for $\chi=80^{\circ}$

curves obtained by him is as symmetrical as those for aegirine, and is fairly different from those obtained by the writer.

\section{Summary}

The intensity of light transmitted through the (010) section of topaz, aegirine and heikolite was measured by a photomultipliergalvanometer set with monochromatic lights of various wave lengths.

In both topaz and aegirine, the intensity curve was found to be in agreement with that calculated from the theoretical equation.

For heikolite, the minimum intensity is considerably strong when nicols are crossed, and as $\chi$ decreases from $90^{\circ}$ to $0^{\circ}$, it gradually decreases until $\chi$ takes the value of $40^{\circ} \sim 50^{\circ}$ and then slightly increases with farther decrease of $\chi$.

The intensity curves obtained from the measurement with crossed nicols were found to show the same behaviour at every $\frac{\pi}{2}$ of the 
angle of rotation and symmetrical behaviour with respect to the positions of maxima and minima. With oblique nicols, the curves showed asymmetrical behaviour but for the measurement with the light of the wave length $668 \mu \mu$. However, as in the case of aegirine, the phase difference between the curve for $\chi$ and that for $-\chi$ is always $\chi$.

\section{Acknowledgements}

The writer wishes to thank Prof. T. Ito, the Institute of Mineralogy, and to Prof. N. Katayama, College of General Education, for their guidance during the present work. He is also indebted to the staff of the Institute of Earth Science and to Mr. K. Nakamura for their assistance in the experiment.

\section{References}

1) Eskola, P. and T. S. Shahlstein: Bull. Comm. Geol. Finland, 92, 89 (1930).

2) Harada, Z.: Jour. Geol. Soc. Jap., 46, 291 (1939).

3) Hori, S.: Jour. Geol. Soc. Jap., 49, 445 (1942).

4) Inuzuka, $H$. and $K$. Kanehara: Beiträge zur Mineralogie von Japan, Neue Folge 1, 98 (1935).

5) Iwao, S.: Jap. Jour. Geol. Geogr., 16, 155 (1939).

6) Katayama, N.: Beiträge zur Mineralogie von Japan, Neue Folge 2, 77 (1937).

7) Katayama, N. and Y. Sato: Jour. Miner. Soc. Jap., 1, 73 (1953).

8) Kinosaki, Y.: Geological Atlas of Korea, 14 (1932).

9) Kinosaki, Y.: Jour. Chosen Mining Ass. (Chosen Kogyokaishi) 18, 187 (1935).

10) Morozewicz, J.: Tschermaks Miner. Petr. Mitt., 38, 210 (1925).

11) Quensel, P. D. . Bull. Geol. Inst. Upsala, 12, 129 (1914).

12) Sato, S.: Report of the first scientific expedition to Manchukuo, sect. 2, part 3, 139 (1936).

13) Vendl, A.: Zeit. Krist., 60, 135 (1924).

14) Yagi, K.: Bull. Geol. Soc. America, 64, 769 (1953).

Manuscript received January 29, 1954. 